\title{
COLLOQUE PISTIS ET FIDES. LE DE OFFICIIS CICÉRONIEN: UNE REFONDATION PHILOSOPHIQUE DE LA FIDES?
}

\author{
Nota del s.c. CARLOS LÉVY (*)
}

(Adunanza del 27 febbraio 2014)

Premessa. - Signori e Signore dell'Accademia, cari colleghi e amici,

mi sarebbe piaciuto presentarmi già in precedenza davanti a voi per comunicarvi tutta la gioia che ha provocato in me l'insigne onore che mi avete accordato. Non ho potuto farlo, e me ne scuso con voi, perché questo è l'anno alla fine del quale andrò in pensione, e ciò ha comportato un aumento del carico di lavoro che non avevo previsto.

Fare parte della vostra prestigiosa Compagnia rappresenta per me più ancora che il riconoscimento di un percorso di insegnante e studioso. Per quanto io possa andare indietro nel tempo con la memoria, l'Italia è stata, ed è, presente nella mia vita in forme diverse. L'Italia degli espatriati, prima di tutto, dal momento che, avendo avuto la fortuna di nascere in una città internazionale, Tangeri, ho conosciuto fin dall'infanzia, io che non sono italiano, il mondo così caloroso e coinvolgente di coloro che si riunivano alla Casa d'Italia, al Club Alpino Italiano o alla Dante Alighieri. E poi l'Italia sognata, quella che ho scoperto leggendo le Bucoliche e le Georgiche, questo paese che mi appariva, quando abitavo tanto vicino alle colonne d'Ercole, come una sorta di trascendenza del Mediterraneo. L'Italia scoperta all'età di vent'anni, con una meraviglia che si rinnovava a ogni istante. L'Italia studiata per tanti anni attraverso i testi di Lucrezio, di Cicerone e di Seneca, per mostrare, contro tanto scetticismo e cattiva fede, che il latino non fu un ostacolo per la filosofia, ma anzi ne costituì la fortuna, perché le permise di raggiungere l'universalità che essa rivendicava. L'Italia universitaria, il paese dove conto il maggior numero di amici, soprattutto all'Università di Milano, con la quale ho il privilegio di collaborare per organizzare, ogni due anni, un convegno nello splendido quadro di Gargnano. La vostra Italia infine, quella di un'Accademia nata da ciò che la Francia ha potuto produrre di meglio, luogo di espressione di tutti i saperi al livello più

(*) Université Paris-Sorbonne, France. E-mail: carlos.levy49@gmail.com 
alto, dove persino nei miei sogni più folli non avrei mai immaginato di poter essere ammesso.

$* * *$

ABSTRACT. - This study intends to follow the semantic evolution of the concept of fides in Cicero's thought, from the rhetorical and oratorical works to the political ones till to the philosophical treatises of the latest years. One can generally say that before 54 b.C. , the date of publication of the De re publica, Cicero, a great user of the concept, at least in the rhetorical field, has a rather confused notion of it, founded on the search much less for its precise sense than for the effectiveness of its use. From this treaty he begins to wonder about its origin: if fides, as he believes, has a natural origin, how to explain its detachment from the world's practices? It is, therefore, to understand what fides' naturalness means; and this is what Cicero tries to do in the philosophical works of the latest period. First of all in the Academica he investigates the place fides occupies at the level of knowledge, in opposition to Lucullus' dogmatic perspective. At the end of this reflection, permeating also the De Finibus and the De Natura Deorum, it comes out confirmed the natural origin of fides, on the condition that you rescue the concept of nature from the Epicurean reconstruction of axiology, founded on sensation and voluptas. This is what Cicero devotes himself to in his very last work, the De officiis, seeking to make of fides the very foundation of iustitia but also trying to reconcile its roots in nature with the variability of the circumstances: that is, giving a less intransigent interpretation of it, while maintaining its ideal value, which finds embodiment in the figure of Regulus.

\section{DE L'ÉLOQUENCE À LA PHILOSOPHIE: L'ÉVOLUTION SÉMANTIQUE DE FIDES}

Fides appartient, comme uoluntas, à ces mots latins qui ont été des notions au champ sémantique vaste et aux frontières imprécises, utilisées comme des évidences à la fois individuelles et collectives, avant que l'arrivée des philosophes, à partir du IIème siècle av. J.C., ne marquât le début d'un questionnement qui aboutira avec Cicéron à la première tentative de formalisation, tendant à transformer ainsi la notion en concept ${ }^{1}$. Dans un premier temps, nous avons été tenté de limiter le sujet, de nous en tenir à l'aboutissement, en nous cantonnant au De officiis. Il nous est vite apparu que cette démarche n'était plus simple qu'en apparence, et qu'il était somme toute préférable de reprendre le problème diachroniquement, sans aucune prétention à l'exhaustivité, mais en nous efforçant de mettre en lumière les étapes les plus importantes de

1 Sur l'ensemble des sens de fides, voir G. Freyburger, Fides. Étude sémantique et religieuse depuis les origines jusqu'à l'époque augustéenne. Paris, 1986. 
cette évolution. Du point de vue méthodologique, nous avons introduit une innovation qui est de ne pas nous contenter d'étudier les occurrences de fides, mais de nous intéresser aussi à ses absences et de chercher à comprendre le pourquoi de celles-ci. C'est en fonction de tout cela que nous avons défini trois orientations, destinées à nous permettre d'analyser les conditions de constitution d'un méta-savoir axiologique en milieu romain:

- la fides de l'orateur et de l'homme politique, océan dans lequel nous ne nous aventurerons qu'avec prudence, dans la mesure où les implications culturelles, idéologiques, anthropologiques et religieuses dépassent largement le cadre de cette étude;

- le temps des questions, qui recouvre les premières œuvres philosophiques cicéroniennes, écrites après la guerre civile;

- enfin le De officiis comme aboutissement non artificiel à la fois d'une expérience et d'un questionnement.

\section{LA FIDES DE L'ORATEUR ET DE L'HOMME POLITIQUE}

Il nous semble que, dans la pratique rhétorique et politique de Cicéron, la fides se caractérise par une tension. Elle est une vertu, autrement dit un élément du processus de perfection de l'individu, dans le contexte d'une tradition ancestrale, celle du mos maiorum, certes bien mal en point dans la réalité, du fait de la crise de la res publica, mais qui restait une référence obligée dans l'éloquence politique, même pour ceux qui avaient compris qu'il fallait passer à autre chose, comme le confirme, entre autres, le discours de César dans la Conjuration de Catilina de Salluste. Elle est même, sous la forme d'une abstraction divinisée, l'un des éléments les plus anciens de la religion civique 2. Politiquement, socialement, la fides cimente la res publica. Mais la fides est aussi, pour l'orateur, le sentiment de confiance qu'il doit faire naître dans le public d'un procès et chez les juges, elle apparaît comme le matériau qu'il lui faut inlassablement travailler. Autant la fides du mos maiorum se veut être, au moins au niveau des représentations, le ciment des relations sociales, une valeur immuable, distinguant le Romain des autres peuples, et à l'intérieur même de la res publica, le bon citoyen des

2 Voir Denys d'Halicarnasse, Ant. II, 75; Tite Live, I, 21, 4; Plutarque, Numa, 16. 
autres, autant la fides recherchée par l'orateur se prête à la manipulation et ne peut prétendre aller au-delà du probabile, autrement dit d'un relatif raisonnable. Essayons donc d'analyser plus profondément ces deux aspects.

La fides est donc une vertu hautement revendiquée par les Romains, une vertu en quelque sorte identitaire qui est censée les différencier de tous les autres peuples, mais la pistis, elle, ne joue qu'un rôle relativement modeste à l'intérieur du système des vertus des doctrines hellénistiques, dans lequel elle n'a jamais eu la place d'une vertu cardinale. Son champ sémantique, très riche chez Platon et chez Aristote, semble s'être considérablement rétréci. En particulier, elle est, pour les Stoïciens, une modalité de la katalèpsis, autrement dit de l'assentiment donné à une représentation «cataleptique», elle apporte de la stabilité à ce que l'on a compris ${ }^{3}$. Chez Épicure, de manière significative, la pistis est présente dans les lettres à Hérodote et à Pythoclès, mais absente de celle à Ménécée et donc de la présentation de l'éthique. De fait, elle paraît surtout avoir été dans cette doctrine une caractéristique épistémologique de la conviction, dont les conséquences éthiques ne paraissent pas avoir été exploitées. Tout au plus est-il dit, dans la Sentence Vaticane 7, qu'il est impossible pour celui qui a commis une faute d'acquérir la pistis de son impunité. En revanche, Cicéron, par souci de ne pas négliger l'idéologie nationale va se trouver conduit à l'évoquer parmi les vertus, mais sans jamais procéder à une intégration systématique. En voici un exemple qui nous semble être caractéristique:

- dans le Pro Murena, 30, autrement dit dans un discours duquel la philosophie n'est pas absente, puisque Caton, incarnation du stoïcisme romain, figure parmi les adversaires du client de Cicéron, l'orateur-philosophe évoque la question des bioi, des genres de vie, et, à l'intérieur même de celle-ci, distingue les vertus personnelles de celles qui permettent d'accéder aux honneurs: des premières, il dit qu'elles valent par elles-mêmes, per se ualent, et il en donne la liste suivante: iustitia, fides, pudor, temperantia. A l'intérieur d'un ensemble encadré par deux vertus reconnues comme canoniques, la justice et la tempérance, on trouve la fides et le pudor, dont on ne peut pas dire qu'elles sont sans équivalents grecs, mais qui ne figu-

3 Voir SVF III, 548. 
rent pas sous cette forme dans des textes philosophiques grecs de l'époque classique. En revanche, cette iunctura est présente chez Épictète et Marc Aurèle, sans qu'on puisse dire si, chez eux, elle relève d'une tradition romaine ou d'un mode d'expression à l'intérieur du stoïcisme tardif, la distinction entre les deux n'étant pas toujours facile à établir". Il n'y a pas nécessairement dans l'énumération de ces quatre vertus substitution de la fides et du pudor au courage et à la prudence, mais reformulation du cadre arétologique en fonction des nécessités de la cause à défendres. Ce qui nous paraît tout particulièrement intéressant ici, c'est que la fides est présentée comme une vertu individuelle, indépendante de toute considération de prestige social, alors même que sa fonction dans l'éthique sociale avait été soulignée bien avant Cicéron. Caton le Censeur ne regrettait-il pas déjà que la fides (frg 58) des ancêtres eût disparu? Et Cicéron lui-même ne cite-t-il pas en Off. III, 104, un vers du poète national Ennius qui en dit long sur le respect, de nature religieuse tout autant que sociale, porté à la Fides dans les premiers siècles de la République?

Cette manière d'entremêler les vertus cardinales avec celles perçues comme essentielles par le peuple romain, qu'il s'agisse de la fides ou de la grauitas, est fréquente chez Cicéron et son fonctionnement apparaît avec une clarté particulière, dans Cat. II, 25. Le consul de 63 commence par évoquer devant son public les qualités auxquelles le sénat romain pouvait être le plus immédiatement sensible: la fides, la constantia, l'bonestas, la continentia. Ce n'est qu'après que, introduite par un denique, survient la liste des vertus traditionnelles: aequitas, temperantia, fortitudo, prudentia. Quel est le sens de cette juxtaposition? Cicéron ne pouvait pas ignorer les liens existant entre les deux listes, il ne s'adresse pas à deux publics différents, il joue sur deux registres, celui de la culture romaine traditionnelle et celui de la culture savante, deux registres que désormais tout Romain appartenant à la classe dirigeante portait en lui. Que la fides soit considérée comme l'essence de la romanité, nous le voyons, en particulier, dans le Pro Flacco, 36, discours où, comme on le sait, Cicéron s'en prend non seulement aux Grecs mais également aux Juifs. Chez les Grecs, dit-il, il

4 Épicure, Hér. 63; Pyth. 85.

5 Pro Caecina 104. Lucilius 30, 1048 Marx. 
n'y a ni firmum consilium, ni testimoni fides ${ }^{6}$. Au moins au niveau des représentations, la fides s'inscrit dans une axiologie cicéronienne, aux accents souvent xénophobes, qui privilégie ce qui est fixe, pesant, car elle est inséparable de la grauitas, insensible au passage du temps. Pas de fides sans grauitas, pas de grauitas sans fides. Dans le domaine du politique, comme dans sa philosophie si marquée par le platonisme, la parole cicéronienne fonctionne selon une double polarité, celle de la fixité, de la Forme ou de la romanité, et celle de la légèreté, de l'inconsistance, commune à beaucoup de peuples, mais incarnée principalement par les Grecs. Dans les Paradoxes des Stö̈ciens ${ }^{7}$, nous voyons comment le héros par excellence aux yeux de Cicéron, Régulus, apparaît comme l'incarnation non seulement de la fides, mais, plus généralement de cette fixité éthique qui, dans un contexte immanentiste, représente la seule résistance possible au flux du temps et à l'irruption des événements: paradox 2, section 16, line 3: “Car ce n'était pas sa grandeur d'âme qui était torturée par les Carthaginois, ni sa fermeté de caractère (grauitas), ni sa loyauté (fides), ni sa constance, ni aucune de ses vertus, ni son âme enfin; protégé par un tel rempart et une telle escorte de tant de vertus, son corps était captif, lui-même, il ne put certainement pas l'être".

Par rapport à cette fides-vertu romaine, la fides que l'orateur essaie de focaliser sur sa personne se trouve, nous l'avons dit, en relation avec le probabile, autrement dit avec ce qui, vrai ou faux, est susceptible d'entraîner l'adhésion ${ }^{8}$. De ce point de vue, le rapprochement le plus significatif se trouve dans les Partitions Oratoires, 5, lorsque l'argument est ainsi défini: probabile inuentum ad faciendam fidem'. Nous reviendrons plus loin sur la relation de fides aux termes par lesquels Cicéron traduit le vocabulaire académico-stoïcien de la persua-

6 Flac. 36: Das enim mihi quod haec causa maxime postulat, nullam gravitatem, nullam constantiam, nullum firmum in Graecis hominibus consilium, nullam denique esse testimoni fidem.

7 Paradox. 2, 16.

8 La définition la plus complète du probabile que l'on trouve chez Cicéron est celle qui est donnée dans Inu. I, 46. Probabile autem est id, quod fere solet fieri aut quod in opinione positum est aut quod babet in se ad haec quandam similitudinem, sive id falsum est sive verum.

9 Part. or. 5: Quid est argumentum? Probabile inventum ad faciendam fidem.

10 Voir infra, p. 68. Du point de vue philosophique, le passage le plus explicite 
sion dans le domaine de la gnoséologi $\mathrm{e}^{10}$. Nous préférons en effet nous arrêter ici sur ce que la rhétorique a pu apporter à Cicéron comme cadre de réflexivité par rapport à la fides. Nous voyons que dans le $D e$ inuentione $^{11}$ la fidentia qui désigne cette forme particulière de fides, qu'est la confiance en soi-même, se trouve intégrée, tout comme la grandeur d'âme, la confiance en soi, l'endurance et la ténacité parmi les formes du courage, en un assemblage qui n'est pas sans rappeler la subdivision stoïcienne des vertus qui nous est donnée par Diogène Laërce. Fidentia est apparemment un terme que Cicéron a forgé pour

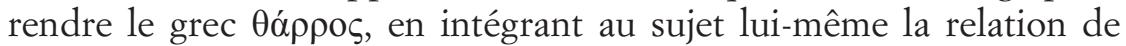
confiance et qu'il ne reprendra, comme nous le verrons plus loin, que quarante ans plus tard, dans les Tusculanes. Pour le reste, le De oratore qui prolonge l'utilisation de la fides à l'intérieur d'un ensemble de vertus, associant les axiologies grecque et romaine ${ }^{12}$, permet néanmoins un approfondissement de la notion, d'une part, en mettant en évidence ses ressorts à travers la célèbre triade, d'inspiration aristotélicienne, associant le probare, le conciliare et le mouere et, d'autre part, en approfondissant son statut de vertu. Sur le premier point, nous renvoyons à ce passage du livre III où Cicéron considère que les trois registres, l'explication, la conciliation, l'émotion sont autant de

établissant une relation entre le probabile et la fides se trouve dans Luc. 35-36: Quod est igitur istuc vestrum probabile? nam si quod cuique occurrit et primo quasi aspectu probabile videtur id confirmatur, quid eo levius?; sin ex circumspectione aliqua et accurata consideratione quod visum sit id se dicent sequi, tamen exitum non babebunt, primum quia is visis inter quae nibil interest aequaliter omnibus abrogatur fides. deinde, cum dicant posse accidere sapienti ut cum omnia fecerit diligentissimeque circumspexerit existat aliquid quod et veri simile videatur et absit longissime $<a>$ vero, $<$ ne $>$ si magnam partem quidem, ut solent dicere, ad verum ipsum aut quam proxime accedant confidere sibi poterunt.

11 Inu. II, 163: Fortitudo est considerata periculorum susceptio et laborum perpessio. eius partes magnificentia, fidentia, patientia, perseverantia. Cf. DL. VII, 92, où, après l'énumération des quatre vertus cardinales nous trouvons ceci: $\dot{\varepsilon} v \varepsilon \grave{v} \delta \varepsilon \imath \delta \dot{\varepsilon} \tau o v ́ \tau \omega v$

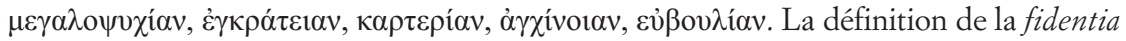
est celle-ci: fidentia est, per quam magnis et honestis in rebus multum ipse animus in se

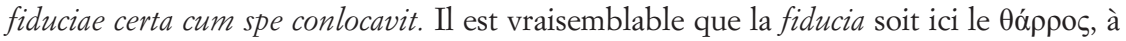
propos duquel voir Stobée Ecl. II, p. 71, 15 W=SVF, III, 106.

12 Voir De or. II, 67: ut de rebus bonis aut malis, expetendis aut fugiendis, bonestis aut turpibus, utilibus aut inutilibus, de virtute, de iustitia, de continentia, de prudentia, de magnitudine animi, de liberalitate, de pietate, de amicitia, de officio, de fide, de ceteris virtutibus contrariisque vitiis dicendum oratori putemus. 
moyens de donner de la fides au discours ${ }^{13}$. Pour ce qui est du second, reprenant une distinction d'Aristote dans sa Rhétorique, Cicéron distingue les vertus intellectuelles de celles qui sont considérées comme politiques et sociales, parmi lesquelles figurent la clémence, la justice, la générosité, la loyauté et le courage ${ }^{14}$. On peut donc dire qu'avant 54 , date de publication du De re publica, Cicéron, grand utilisateur au moins rhétorique du concept de fides en a une notion assez confuse, fondée beaucoup moins sur la recherche de son sens précis que sur celle de l'efficacité de son utilisation. De toutes les définitions, la plus précise est celle que nous trouvons dans les Partitions Oratoires ${ }^{15}$ où la fides est dite être une firma opinio, ce qui, d'un point de vue philosophique, constitue une contradiction dans les termes, puisque, si l'on prend en particulier la définition stoïcienne de la doxa, celle-ci est définie soit comme l'assentiment à une proposition fausse, soit comme l'assentiment faible à une proposition vraie ${ }^{16}$. Dans le premier cas, la fides est épistémologiquement et axiologiquement dévalorisée, dans le

13 De Or. III, 104: Id desideratur omnibus eis in locis, quos ad fidem orationis faciendam adbiberi dixit Antonius, vel cum explanamus aliquid vel cum conciliamus animos vel cum concitamus, sed in hoc, quod postremum dixi, amplificatio potest plurimum, eaque una laus oratoris est [et] propria maxime.

14 De Or. II, 343: Virtus autem, quae est per se ipsa laudabilis et sine qua nibil laudari potest, tamen babet pluris partis, quarum alia est <alia> ad laudationem aptior; sunt enim aliae virtutes, quae videntur in moribus bominum et quadam comitate ac beneficentia positae; aliae, quae in ingeni aliqua facultate aut animi magnitudine ac robore; nam clementia, iustitia, benignitas, fides, fortitudo in periculis communibus iucunda est auditu in laudationibus; omnes enim bae virtutes non tam ipsis, qui eas babent, quam generi hominum fructuosae putantur. Sur ce point, voir J. Wisse, Ethos and Pathos from Aristotle to Cicero, Amsterdam, 1989, p. 11!-121.

15 Part Or. 9, 8: Quoniam fides est firma opinio, motus autem animi incitatio aut ad voluptatem aut ad molestiam aut ad metum aut ad cupiditatem (tot enim sunt motus genera, partes plures generum singulorum), omnem collocationem ad finem accommodo quaestionis.

16 Voir l'exposé de la doctrine de Zénon dans Lib. Acad. I, 41: Quod autem erat sensu comprensum id ipsum sensum appellabat, et si ita erat comprensum ut convelli ratione non posset scientiam, sin aliter inscientiam nominabat; ex qua existebat etiam opinio, quae esset imbecilla et cum falso incognitoque communis. Sur cette question voir C. Lévy, "Le concept de doxa des Stö̈ciens à Philon d'Alexandrie, essai d'étude diachronique", dans J. Brunschwig et M. C. Nussbaum eds, Passions and Perceptions. Studies in Hellenistic Philosopby of Mind, Cambridge 1993, p. 250-284. 
second, elle ne peut pas être firma, malgré la vérité objective de son contenu. Il y a donc jusque là, pour l'essentiel, une pragmatique de la fides, dont le soubassement théorique demeure des plus confus, mais qui a pour finalité d'exploiter dans le cadre d'un procès les faiblesses rationnelles et émotives de l'auditeur, de façon à installer en lui la thèse que l'on veut faire triompher.

C'est donc dans le De re publica, premier traité d'inspiration philosophique, que l'interrogation sur l'origine et les enjeux de cette vertu commence à apparaitre. Significative est la question que l'on trouve dès les premières lignes du traité: unde iustitia, fides, aequitas? Il ne s'agit plus d'invoquer les vertus du mos maiorum, mais d'en rechercher le fondement. Cette démarche passe aussi par une approche étymologique. C'est en effet dans le De re publica que nous trouvons pour la première fois, en IV, 7, la célèbre étymologie qui lie la fides à l'effectivité du discours ${ }^{17}$ : Fides enim nomen ipsum mibi videtur habere, cum fit, quod dicitur. Celle-ci, que l'on dit être de type varronien, sera reprise par lui dans le premier livre du De officiis, I, 23, sous une forme à peine différente, et avec une identification stö̈cienne cette fois clairement affirmée ${ }^{18}$. Loin d'avoir ici la valeur d'un simple jeu de mots, l'utilisation de cette étymologie exprime le désir d'une fondation naturaliste de la fides. Le langage ayant dans une perspective stö̈cienne un fondement naturel, la fides se trouve ainsi enracinée dans une correspondance première entre les mots et les choses. Malgré l'état lacunaire du De re publica, et en particulier de son troisième livre, nous pouvons deviner la place qu'y tenait la problématique de la fides. Dans l'antilogie censée reproduire les deux discours romains de Carnéade, lors de l'ambassade des philosophes en 155, le juste dont Philus, s'efforçant de montrer l'absurdité même de l'idée de justice, va montrer qu'il n'a aucune place dans le monde tel qu'il est, est qualifié de vir aequissimus, summa iustitia, singulari fide. Il sera supplicié, souffrira mille morts, alors que l'injuste sera lui couvert d'honneurs et vivra dans l'aisance que lui vaudront ses méfaits. La grande question qui se pose alors est celle-ci: si, comme semble l'indiquer le langage, ou en tout cas l'inter-

17 Rep. IV, 7.

18 Off., I, 23: Ex quo, quamquam hoc videbitur fortasse cuipiam durius, tamen audeamus imitari Stoicos, qui studiose exquirunt, unde verba sint ducta, credamusque, quia fiat, quod dictum est appellatam fidem. 
prétation qu'en donne Cicéron, la fides a une origine naturelle, comment se fait-il qu'elle puisse se révéler si décalée par rapport aux pratiques du monde? Que signifie alors la nature?

Cicéron se trouve donc en quelque sorte contraint d'entrer dans l'exploration de ce concept, autrement dit de tenter de comprendre ce que signifie la naturalité de la fides, ce qu'il va faire, dix ans après le $D e$ re publica, à travers les trois parties de la philosophie hellénistique.

En premier lieu, la théorie de la connaissance. Ce n'est pas le moindre des éléments d'intérêt que comportent les Académiques que d'y voir la place qu'y tient la fides comme concept gnoséologique.

En I, 41, un élément montre la force de la fides dans la pensée de Cicéron. Décrivant le processus de la représentation cataleptique, caractérisée par l'évidence et entraînant le sujet vers l'assentiment comme en le tirant pars les cheveux, pour reprendre la célèbre métaphore de Chrysippe ${ }^{19}$, Cicéron dit de Zénon qu'il n'ajoutait pas foi à toutes les représentations, mais seulement à celles qui représentaient avec une clarté singulière les objets soumis à la vision. Alors que le terme de fides aurait pu être utilisé comme équivalent de pithanon, comme c'était le cas dans le domaine de la rhétorique, pour désigner une représentation donnant le sentiment de la vérité mais qui ne serait pas pour autant vraie, Cicéron lui donne un sens très fort, en faisant le parfait synonyme d'adsensio ou d'adsensus. Le fondement de cette adhésion à la représentation cataleptique est expliqué tout de suite après: la fides du sujet par rapport à ces représentations s'explique par le fait que la nature a donné en elles "comme une norme de science et son propre principe". La fides est donc, au niveau de la connaissance, l'équivalent de ce que sont la conciliatio et la commendatio, traductions latines d'oikeiôsis, dans le monde de l'éthique. Tout comme l'individu qui naît est confié à sa nature, la représentation cataleptique est l'espace où se noue une relation de confiance entre le sujet et le nature, relation qui conditionne tout le fonctionnement de la raison. Si la représentation cataleptique n'existe pas, quelle confiance méritera une démonstration probante? Tout comme la recherche du telos n'a de sens dans le stoïcisme que par rapport à l'oikeiôsis initiale que l'homme, seul être rationnel, partage avec tous les êtres animés, toute argumentation

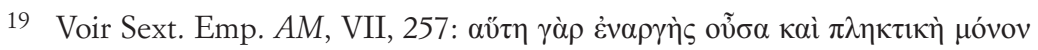

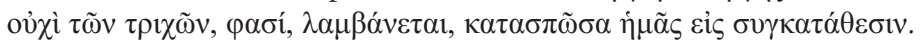


rationnelle renvoie à la fides première. Dans une telle perspective, ce n'est pas la connaissance seule qui est menacée par l'absence de celleci, mais l'action et toutes les valeurs morales. Enlever aux représentations la fides que leur donne l'évidence naturelle, c'est donc, dans la perspective dogmatique défendue par Lucullus, tout simplement ruiner la vie. Or, on ne semble pas avoir remarqué que si le terme de fides est employé un grand nombre de fois par Lucullus, il n'apparaît qu'une seule fois dans le discours de Cicéron, et encore dans un contexte littéraire qui lui enlève toute valeur philosophique, $\mathbb{S} 89$. Si l'on considère les discours de Lucullus et celui de Cicéron comme une disputatio in utramque partem, le cœur de celle-ci est donc de savoir si la relation première de fides entre l'être humain et le monde dans la représentation est nécessaire à la vie ou non. Le Cicéron du dialogue pense que l'on peut très bien vivre sans.

Je m'arrêterai quelques instants sur un des passages du Lucullus où apparaît le mieux la complexité de l'emploi cicéronien de la fides: dum aspectus ipse fidem faciat sui iudicii. Lorsque nous voyons un objet dans la pénombre et que nous ne sommes pas sûrs de l'identifier, nous multiplions les essais jusqu'à ce que "la vision elle-même provoque notre confiance en son jugement", (19, traduction de J. Kany Turpin). Un passage très proche chez Sextus Empiricus, $\$ 258$, permet de mieux appréhender la spécificité de l'emploi cicéronien. Ce que dit Sextus, c'est que ceux qui pratiquent ces ajustements font comme si la pistis katalepseôs (crédibilité de l'appréhension) reposait sur la qualité de la représentation. En réalité les nuances sont assez différence. "Fidem facere" est l'expression qui est employée dans les procès pour signifier emporter l'adhésion, acception technique qu' atteste la Rhétorique à Hérennius. Iudicium utilisé là où le grec emploie katalèpsis confirme ici la métaphore. Tout comme en traduisant oikeiôsis par conciliatio et commendatio, Cicéron avait exprimé le naturalisme stoïcien en termes de pratiques sociales romaines, ici, la relation naturelle du sujet de la connaissance à l'objet se trouve formulée à travers la métaphore du procès. C'est l'image qui devient l'avocat en quelque sorte du lekton qu'elle porte en elle. Dans une telle métaphore qu'on sera en droit de juger peu appropriée, c'est en quelque sorte l'oikeiôsis de Cicéron à sa persona d'orateur philosophe qui se joue aussi.

J'en viens maintenant aux traités philosophiques qui ont précédé le De officiis et tout particulièrement au De finibus. La différence par rapport aux Academica me semble être celle-ci. Dans le premier dia- 
logue, le débat oppose Lucullus, qui proclame la pertinence du modèle de l'oikeiôsis (39) aussi bien dans le domaine de la connaissance que dans celui de l'action: "car de même qu'il est impossible à l'être animé de ne pas tendre vers ce qui lui paraît approprié à sa nature, de même il lui est impossible de ne pas approuver ce qui se présente avec évidence”, à quelqu'un Cicéron, qui, dans le domaine de la connaissance au moins, rejette ce modèle. D'un côté, nous l'avons vu, une pensée de la fides, de l'autre, une pensée qui l'ignore. La situation est différente dans le De finibus, puisque les trois livres dogmatiques sont tous les deux fondés sur l'affirmation d'une oikeiôsis initiale à la nature. Le problème qui se pose n'est donc pas celui d'un lien naturel initial, mais celui de l'interprétation du lien initial. Que devient la fides selon que l'oikeiôsis est épicurienne, stoïcienne ou académico-péripatéticienne? La première remarque est l'absence de la fides dans le livre III, pour laquelle je n'ai pas d'explication pleinement satisfaisante. Tout au plus est-il possible de dire que la problématique du telos telle qu'elle est développée par Caton, assez strictement calquée sur la terminologie stoïcienne, ne laissait que peu de place à la question de la fides. On notera également que, dans les Tusculanes, où Cicéron poursuit un itinéraire personnel de lutte contre les passions et d'interrogation sur le devenir de l'âme après la mort, il n'y a pas non plus d'occurrence de fides, celle-ci étant convertie en fidentia, autrement dit en expression d'une raison qui a confiance en elle-même. On pourrait être tenté de comprendre fidentia comme un équivalent de authadeia si ce mot n'avait pas un sens nettement négatif, il faut donc penser à quelque chose comme megalopsuchia. Quoi qu'il en soit, Cicéron ne saisit pas l'occasion qui lui était offerte de détacher la fides du naturalisme et de la rattacher au moins occasionnellement à une pensée de la transcendance. En revanche, la fides est bien présente dan le débat entre l'Epicurien Torquatus et l'Académicien Cicéron. Pour Torquatus, la fides dans les sens, différence de la relation stö̈cienne à la sensation en ceci qu'elle est le constat physique de leur exactitude et non la mise en relation du sujet avec le logos universel, fonde la uera ratio dont il affirme, 52, qu'elle convie les hommes qui sont sains d'esprits à la iustitia, aequitas, fides. Dans la mesure où cette vertu est préservée, Cicéron pourrait s'en estimer satisfait, mais ce n'est pas le cas. Au contraire, plusieurs passages du livre II sont consacrés à démontrer que, pas plus que les autres valeurs, la fides ne peut se construire sur l'intérêt. Pour lui, affirmer qu'elle a une origine naturelle, cela signifie qu'elle n'est 
pas un produit dérivé de l'intérêt et du plaisir. Donc le fait que, comme il le reconnaît lui-même, les Epicuriens soient des êtres fideles ne change rien à son refus de considérer la fides comme la résultante d'un certain nombre de forces toutes de caractère hédoniste. Le fait que la philosophie puisse associer étroitement deux termes aussi violemment antagonistes dans le mos maiorum que la fides et la uoluptas lui posait un problème majeur qui était celui de ne pas créer en lui de rupture entre la réflexion philosophique et son identité de consulaire romain, d'où le jeu du dialogue à plusieurs personnages qui lui permettait à la fois d'exprimer des pensées peu conformes à la tradition et de prendre une distance au moins littéraire par rapport à elles.

C'est ce qui apparaît assez clairement dans le De natura deorum, où la Fides n'est envisagée que comme une abstraction divinisée, comme cela était déjà le cas dans le De legibus, réalité dans laquelle le Stoïcien Balbus pouvait voir la perception populaire d'un aspect du Dieu logos, donc de l'enracinement de la Fides dans la nature, et donc de la capacité du peuple romain à percevoir intuitivement ce qu'était la réalité des choses. Cependant, l'Académicien Cotta, dont Cicéron devrait se sentir proche, mais par rapport auquel il prend ses distances, utilise tout l'arsenal de sa dialectique pour démontrer qu'à partir du moment où on entre dans ce processus de divinisation, il n'y a pas de raison de s'arrêter et que, les sorites aidant, on arrivera à diviniser les objets les plus vulgaires.

Lorsque Cicéron termine son programme de réflexion tripartite sur la philosophie, il peut en tirer les conclusions suivantes:

- la fides peut être considérée comme naturelle, mais cette origine n'échappe pas à la critique des sceptiques;

- à supposer qu'elle soit naturelle, que faut-il entendre par nature et comment la faire échapper à la reconstruction par les Epicuriens de toute l'axiologie à partir de la sensation et de la uoluptas.

Ajoutons que la guerre civile, forme ultime de la ruine du lien social, rendait encore plus urgente de revisiter et de reconstruire un concept qui, dans la violence des luttes armées puis de la dictature de César pouvait apparaître comme définitivement obsolète. De l'état d'esprit qui pouvait être le sien face à de tels désastres, je ne donnerai qu'un seul exemple, tiré d'une lettre à Sulpicius, d'avril 49, avant même donc que la guerre n'éclatât: book 4, letter 1, section 2, line 3: Res vides quo modo se habeat: orbem terrarum imperiis distributis ardere bello, urbem sine legibus, sine iudiciis, sine iure, sine fide relictam direptioni et incen- 
diis. La ville est sans droit et sans fides, il appartient donc au philosophe, à défaut de pouvoir assumer un pouvoir dont il continuait à rever, de veiller en quelque sorte à la reconstruction conceptuelle. Ce n'est donc un hasard si, de manière quasiment obsessionnelle, la fides est présente à chaque page d'un traité, le De officiis, dont il convient peut-être de rappeler qu'il ne prétend qu'être une éthique du progressant, fondée sur la plus grande probabilité, encore qu'il entretienne des liens très étroits avec la réflexion téléologique. La première avancée que je signalerai est le fait que cette fois-ci la place de la fides dans l'organisation des vertus se trouve clairement définie. L'un des apports de Panétius, dont Cicéron s'inspire dans les deux premiers livres de son traité, fut de reformuler la classification quadripartite des vertus. La pistis figurait-elle parmi cellesci, nous n'avons pas de moyen de le savoir. Ce que nous pouvons dire, en revanche, c'est que, dès le début de son ouvrage Cicéron procède à cette restructuration en donnant comme vertus fondamentales la

1) perspicientia veri sollertiaque, qui correspond à la phronèsis;

2) la vertu sociale qui se substitue à la justice, vertu consistant in bominum societate tuenda tribuendoque sum cuique et rerum contractarum fide;

3) le courage redéfini en: in animi excelsi atque invicti magnitudine ac robore;

4) la sophrôsunè, qui devient le decorum, prepon, défini comme: in omnium, quae fiunt quaeque dicuntur ordine et modo, in quo inest modestia et temperantia.

La fides n'est plus une vertu plus ou moins gyrovague, au gré des énumérations cicéroniennes, elle apparaît non seulement comme une vertu canonique, mais comme le fondement même de la iustitia, je fais allusion à I, 23: "Fundamentum autem est iustitiae fides, id est dictorum conventorumque constantia et veritas". Contrairement à ce qu'a affirmé A. Dyck en une formule particulièrement malheureuse, "an ad hoc invention", mais bien au contraire l'aboutissement d'une réflexion approfondie. Le fait que la même expression soit utilisée en 42 à propos de la distribution proportionnelle n'induit aucune contradiction, elle est au contraire la confirmation que la justice a été présentée comme une superstructure reposant sur ces trois piliers que sont le maintien de la société, la réponse distributive et le respect des engagements. A partir de là, va se nouer une contradiction qui me semble être au cœur du traité.

Cependant, tout en donnant ce statut principiel à la fides, Cicéron reconnaît qu'elle ne correspond pas à une obligation figée, affirmant 
que "tout cela change avec les circonstances, mais le devoir change aussi et n'est pas toujours identique" (31). Face au risque de relativisme induit par une telle affirmation, il s'agit donc de trouver une norme permettant d'affronter éthiquement le changement: "il ne faut donc pas tenir les promesses qui peuvent être nuisibles à qui tu les as faites, et d'autre part si ces promesses te font plus de tort qu'elles ne profitent à qui tu les as faites, il n'est pas contraire au devoir de préférer le plus grand nombre au moindre".

Ce bon usage de la fides, conciliant son enracinement naturel avec la prise en compte de la variabilité des circonstances, au nom d'une conception éthique rigoureuse et en précisant bien que la fides est la fidélité aux sentiments et non aux paroles renforce l'aspect d'éthique provisoire revendiqué au début du traité.

Mais, par ailleurs, omniprésente, la figure de Régulus, hautement revendiquée par Cicéron, vient contredire cette plasticité de la fides à l'intérieur même du cadre éthique. Régulus, c'est précisément celui qui s'est refusé à ce genre de solution probabiliste. Si l'on prend la règle "il ne faut donc pas tenir les promesses...", il va de soi qu'elle ne peut servir de justificatif à son attitude qui est celle de l'absolu de la fides, la promesse devant être réalisée quel que soit l'environnement.

Ces deux positions me semblent transférer à l'intérieur même de la romanité le débat qui avait opposé, si l'on en croit Cicéron, Diogène de Babylone et Antipater de Tarse, le premier partisan d'une éthique de la casuistique, le second professant une sorte d'impératif catégorique avant la lettre. Dans le débat historico-philosophique, Cicéron choisit nettement Antipater contre Diogène, dans l'économie de son propre livre, il concilie l'exaltation de la figure de Régulus qui devient par ellemême fondatrice de la fides romaine et une conception, disons plus souple, de tout cela. Y a-t-il une logique à tout cela? Il me semble que c'est le cas, si on accepte de se situer dans une temporalité stoïcienne autrement dit circulaire. Dans la lettre 90 sur les débuts de l'humanité, Sénèque raconte qu'au début les hommes étaient sages et heureux, mais de manière non réflexive, si bien qu'ils laissaient à ceux qui venaient après eux la charge de passer de la vertu spontanée à la véritable vertu, celle qui passe par la reconstruction du moi après l'épreuve du réel. A cette conception générale, Cicéron apporte quelques modulations:

- pour un romain, jusqu'à Cicéron précisément, la question générale des origines ne se pose pas, seule compte l'origine de la ville, comme l'indique la formule $a b$ urbe condita; 
- Rome n'est pas, à ses yeux, une cité comme les autres mais une sorte de peuple élu de l'immanence puisque sa législation religieuse au moins est identifiée à la loi naturelle.

Dans ces conditions, les exemples de Régulus et des premiers romains pratiquant une fides sans faille, loin d'être de simples illustrations deviennent fondamentaux, en ce sens qu'ils permettent d'échapper à la fois à la dialectique néoacadémicienne et à la pensée du plaisir. La prise en compte de la temporalité circulaire et de la spécificité de Rome devient l'élément qui permet d'esquiver les arguments utilisés par les uns et par les autres. Dans le même temps, l'exemple de Régulus ne peut être reproduit en réflexivité que par le sage. Mais la morale de Cicéron n'étant que celle du progressant, le probabile de cette fides repensée permet d'offrir une version moins intransigeante de celle-ci, tout en sauvegardant l'idéal.

J'ai souvent dit que, dans l'état actuel de nos textes, il convient de considérer Cicéron, à cause des Tusculanes, comme le premier précurseur du moyen platonisme. Je dois cependant reconnaître, qu'en ce qui concerne la fides, il a gardé tout au long d'une vie qui fut fort longue une attitude marquée par le naturalisme et, tout particulièrement par le naturalisme stoïcien. Il n'y a nulle part dans son œuvre une ouverture vers un dialogue personnel avec Dieu, une ouverture vers la transcendance, préparant en quelque sorte ce qui allait advenir. En ce sens, je dois dire que, si dans d'autres domaines de la langue et de la pensée son intervention fut décisive, en ce qui concerne la "fides" son apport à notre concept moderne de foi fut...modeste.

\section{TEXTES}

1) Pro Murena 30:

Ceterae tamen virtutes ipsae per se multum valent, iustitia, fides, pudor, temperantia; quibus te, Servi, excellere omnes intellegunt.

2) Cat. II, 25:

Ex hac enim parte pudor pugnat, illinc petulantia; hinc pudicitia, illinc stuprum; hinc fides, illinc fraudatio; hinc pietas, illinc scelus; hinc constantia, illinc furor; hinc honestas, illinc turpitudo; hinc continentia, illinc libido; hinc denique aequitas, temperantia, fortitudo, prudentia, virtutes omnes certant cum iniquitate, luxuria, ignavia, temeritate, cum vitiis omnibus. 
3) Flac., 36:

Das enim mihi quod haec causa maxime postulat, nullam gravitatem, nullam constantiam, nullum firmum in Graecis hominibus consilium, nullam denique esse testimoni fidem.

4) Part. or., 5:

\{C.F.\} Quid est argumentum?

\{C.P.\} Probabile inventum ad faciendam fidem.

6.1

\{C.F.\} Quomodo igitur duo genera ista dividis?

\{C.P.\} Quae sine arte putantur, ea remota appello, ut testimonia.

5) De inu., II, 163:

Fortitudo est considerata periculorum susceptio et laborum perpessio. eius partes magnificentia, fidentia, patientia, perseverantia.

6) Rep., IV, 7:

Fides enim nomen ipsum mihi videtur habere, cum fit, quod dicitur.

7) Acad., I, 41:

visis non omnibus adiungebat fidem sed is solum quae propriam quandam haberent declarationem earum rerum quae viderentur; id autem visum cum ipsum per se cerneretur, comprehendibile - feretis haec?' \{ATT.\} 'nos vero' inquit; 'quonam enim alio modo $\kappa \alpha \tau \alpha \lambda \eta \pi \tau$ òv diceres?

8) Luc., 38:

ut enim necesse est lancem in libram ponderibus inpositis deprimi sic animum perspicuis cedere. nam quo modo non potest animal ullum non adpetere id quod adcommodatum ad naturam adpareat (Graeci id oîkeĩov appellant), sic non potest obiectam rem perspicuam non adprobare.

9) Fam., IV, 1:

Res vides quo modo se habeat: orbem terrarum imperiis distributis ardere bello, urbem sine legibus, sine iudiciis, sine iure, sine fide relictam direptioni et incendiis.

10) Off., I, 15:

Aut enim in perspicientia veri sollertiaque versatur aut in hominum societate tuenda tribuendoque suum cuique et rerum contractarum fide aut in animi excelsi atque invicti magnitudine ac robore aut in omnium, quae fiunt quaeque dicuntur ordine et modo, in quo inest modestia et temperantia.

11) Off., I, 23:

Fundamentum autem est iustitiae fides, id est dictorum conventorumque constantia et veritas"

12) Off., I, 31:

Sed incidunt saepe tempora, cum ea, quae maxime videntur digna esse iusto homine, eoque quem virum bonum dicimus, commutantur fiuntque contraria, ut reddere depositum, [Letiamne furioso?]」 facere promissum quaeque pertinent ad veritatem et ad fidem; ea migrare interdum et non servare fit iustum. Referri enim decet ad ea, quae posui principio fundamenta iustitiae, primum ut ne cui noceatur, deinde ut communi utilitati 
serviatur. Ea cum tempore commutantur, commutatur officium et non semper est idem. Potest enim accidere promissum aliquod et conventum, ut id effici sit inutile vel ei, cui promissum sit, vel ei, qui promiserit.

13) Off., III, 44:

Itaque praeclarum a maioribus accepimus morem rogandi iudicis, si eum teneremus, QVAE SALVA FIDE FACERE POSSIT. 